\title{
Optimal information rate of secret sharing schemes on trees
}

\author{
László Csirmaz and Gábor Tardos
}

\begin{abstract}
The information rate for an access structure is the reciprocal of the load of the optimal secret sharing scheme for this structure. We determine this value for all trees: it is $(2-1 / c)^{-1}$, where $c$ is the size of the largest core of the tree. A subset of the vertices of a tree is a core if it induces a connected subgraph and for each vertex in the subset one finds a neighbor outside the subset. Our result follows from a lower and an upper bound on the information rate that applies for any graph and happen to coincide for trees because of a correspondence between the size of the largest core and a quantity related to a fractional cover of the tree with stars.
\end{abstract}

Keywords. Secret sharing scheme; information rate; graph; fractional packing and cover; entropy method.

AMS classification numbers. 05B40, 05C85, 94A60, 94A62, 94A17

\section{INTRODUCTION}

Secret sharing schemes has been investigated in several papers, for an extended bibliography see [14]. Such a scheme with $n$ participants is a joint distribution of $n+1$ discreet random variables, one called the secret, the rest being the shares of the participants. An access structure designates certain subsets of the participants as qualified leaving the rest of the subsets unqualified. A secret sharing scheme for an access access structure has to satisfy that one can recover the secret with probability 1 from the shares of any qualified subset of the participants but the secret should be statistically independent from the collection of shares belonging to an unqualified subset.

In this paper we deal with access structures based on graphs. The scheme is based on the graph $G$ if the participants are the vertices, and unqualified subsets are the independent sets. This makes the endpoints of the edges the minimal qualified subsets. We simply call a secret sharing scheme for the access structure based on a graph $G$ a secret sharing scheme on $G$.

The load of a scheme is measured by the amount of information the most heavily loaded participant must remember for each bit in the secret. Formally, this is $\max _{i}\left(H\left(S_{i}\right)\right) / H(\xi)$, where $S_{i}$ is the share of participant $i, \xi$ is the secret and $H$ denotes entropy. We assume $H(\xi)>0$. For a graph $G$ the information complexity of $G$, denoted as $\sigma(G)$, is the infimum of the loads of all secret sharing schemes on $G$. The information rate, usually denoted as $\rho(G)$, is simply $\rho(G)=1 / \sigma(G)$, the inverse of this value. The notation $\sigma(G)$

Central European University and University of Debrecen. Research was partially supported by grant NKTH OM-00289/2008 and the "Lendület" project.

School of Computing Science, Simon Fraser University, Burnaby, BC and Rényi Institute of Mathematics, Budapest Research was partially supported by NSERC Discovery grant, the Hungarian OTKA grants T-046234, AT-048826, NK-62321 and the "Lendület" project. for the complexity of the scheme was introduced in [10]. The information rate of graphs has been investigated in several papers, see [9] for the rate of graphs with at most six vertices and also (among other works) [2], [3], [4], [5], [6], [7], [13].

In [13] Stinson describes a general secret sharing construction, which, when applied to graphs, gives the upper bound $(d+1) / 2$ for the complexity of graphs with maximum degree $d$. Blundo et al. in [2] constructed an infinite family of graphs for each $d$ for which Stinson's bound is tight. The $d=2$ case is fully settled in [3]: the information complexity of paths and cycles is $3 / 2$ except for $P_{2}, P_{3}, C_{3}$ and $C_{4}$, when it is 1. The information complexity of the $d$-regular $d$-dimensional hypercube is exactly $d / 2$, see [8]. Our paper is the first one which determines the information complexity and information rate of graphs in a large and natural family, namely, for trees.

To state our result we need the notions of core and star cover rate of an arbitrary graph.

Definition 1.1 We call a subset $X$ of the vertices of a graph $G$ a core of $G$ if it induces a connected subgraph and one can find a neighbor $x^{\prime} \notin X$ of each $x \in X$ such that $x$ is the only neighbor of $x^{\prime}$ among the vertices in $X$ and $\left\{x^{\prime} \mid x \in X\right\}$ is an independent set.

A fractional star packing in a graph $G$ is a collection of star subgraphs of $G$, each with an associated positive weight. The weight of a vertex or an edge in a fractional star packing is the total weights associated to stars containing that vertex or edge, respectively. The star cover rate of $G$ is the infimum (minimum) of the maximal vertex weights among all fractional star packings with each edge having weight at least 1 .

If the weights in a fractional star packing are integral we speak of star packing and we say a vertex or edge is covered $k$ times if its weight is $k$.

Notice that when $G$ is a tree a subset $X$ of its vertices is a core if it induces a connected subgraph and each $x \in X$ has a neighbor outside $X$.

Theorem 1.2: Let $G$ be a graph, let $c=c(G)$ be the maximum size of a core of $G$ and let $s=s(G)$ be the star cover rate of $G$. For the information complexity $\sigma(G)$ of $G$ we have

$$
2-1 / c \leq \sigma(G) \leq s .
$$

Note that the second inequality of this theorem comes from Stinson [13]. We state it here for completeness. Both the lower and the upper bounds are often useful, but they are not tight in general. The graph $\Delta$ depicted in Figure 1 has only one vertex cores, its information complexity is $3 / 2$ and its star cover rate is $5 / 3$. Thus we have strict inequalities in

$$
2-1 / c(\Delta)<\sigma(\Delta)<s(\Delta) .
$$




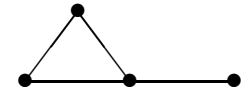

Fig. 1. A graph with different information complexity, maximum core size and star packing rate

For trees, however, our lower and upper bounds coincide and we can even compute this value efficiently.

Theorem 1.3: Let $G$ be a tree, let $c=c(G)$ be the maximum size of a core of $G$ and let $s=s(G)$ be the star cover rate of $G$. For the information complexity $\sigma(G)$ of $G$ we have

$$
2-1 / c=\sigma(G)=s .
$$

One can compute $c$ and thus $\sigma(G)$ and the information rate $\rho(G)$ in linear time. Furthermore, a linear secret sharing scheme exists on $G$ that achieves optimal load $2-1 / c$. In this scheme the shares are vectors of length $2 c-1$ over a finite field, the secret is a vector of length $c$ and these are computed applying linear maps to a uniform random vector of some fixed length less than $n c$, where $n$ is the number of vertices in $G$. The actual matrices providing the linear maps can be found in time linear in the output size.

In Section $\Pi$ we prove the lower bound part of Theorem 1.2 using the entropy method, see [7], [8]. Note that the upper bound comes from Stinson [13].

We prove the equalities of Theorem 1.3 in section [III by proving that $s(G)=2-1 / c(G)$ if $G$ is a tree.

Finally in Section IV we prove the algorithmic assertions of Theorem 1.3 .

\section{INFORMATION COMPLEXITY OF GENERAL GRAPHS}

In this section we show that the information complexity of an arbitrary graph is at least $2-1 / c$ where $c$ is the size of the largest core in $G$. This proves the $2-1 / c \leq \sigma(G)$ part of Theorem 1.2

The proof uses the entropy method, see, e.g. [7], [8]. For the sake of completeness we sketch how this method works. Consider any secret sharing scheme for an arbitrary access structure. For any subset $A$ of the participants we define $f(A)$ to be the normalized entropy of the shares belonging to the participants in $A$, namely

$$
f(A)=\frac{H\left(\left\{S_{v} \mid v \in A\right\}\right)}{H(\xi)},
$$

where $S_{v}$ is the share of participant $v$ and $\xi$ is the secret. Note that our goal is to lower bound the load of the scheme, which is $\max _{v} f(\{v\})$.

Using the standard (Shanon-type) information inequalities we have

(a) $f(\emptyset)=0$,

(b) $f(A) \geq f(B)$ when $A \supseteq B$ (monotonicity) and

(c) $f(A)+f(B) \geq f(A \cup B)+f(A \cap B)$ (submodularity).

Using the definition of the secret sharing schemes we further have

(d) $f(A) \geq f(B)+1$ when $A \supseteq B, A$ is qualified while $B$ is not (strict monotonicity) and

(e) $f(A)+f(B) \geq f(A \cup B)+f(A \cap B)+1$ when $A, B$ are qualified while $A \cap B$ is not (strict submodularity).
The entropy method involves proving a lower bound for $\max _{v} f(\{v\})$ for any $f$ satisfying inequalities (a)-(e). In our case we want to show that there is always a vertex $v$ with $f(\{v\}) \geq 2-1 / c$ and this clearly follows from the following lemma.

Lemma 2.1: Let $X$ be a core of the graph $G$, and let $f$ be a real valued function defined on the subsets of the vertices of $G$ satisfying properties (a)-(e). Then

$$
\sum_{v \in X} f(\{v\}) \geq 2|X|-1 .
$$

Proof: First observe that the statement is trivial if $|X| \leq$ 1. We can therefore assume $|X| \geq 2$. We use the "independent sequence lemma" from [1], [8] that ensures

$$
f(X) \geq|X|+1 .
$$

Using this inequality it is enough to prove

$$
\sum_{v \in X} f(\{v\}) \geq f(X)+|X|-2 .
$$

We prove this latter inequality for all subsets $X$ that induce a connected subgraph, not only for cores. We use induction on the number of the vertices in $X$. The base case $X=\{v, w\}$ of (1) simplifies to

$$
f(\{v\})+f(\{w\}) \geq f(\{v, w\})
$$

which is subadditivity and a consequence of properties (a) and (c).

Now suppose $X$ induces a connected subgraph and it has at least three vertices. Let us pick a vertex $v \in X$ such that $Y=X-\{v\}$ also induces a connected subgraph. Note that such a vertex $v$ always exists. Let $w$ be a vertex in $Y$ connected to $v$. Neither $\{v, w\}$ nor $Y$ is an independent set (we use $|X| \geq 3$ here), but their intersection $\{w\}$ is independent, thus unqualified. Property (e) gives

$$
f(\{v, w\})+f(Y) \geq f(X)+f(\{w\})+1 .
$$

Also, $f(\{v\})+f(\{w\}) \geq f(\{v, w\})$ by subadditivity, which yields

$$
f(\{v\})+f(Y) \geq f(X)+1 .
$$

The induction hypothesis for $Y$ finishes the proof of (1) and also the proof of the lemma.

\section{INFORMATION COMPLEXITY OF TREES}

In this section we show the equalities stated in Theorem 1.3 They follow from Theorem 1.2 and the following lemma. To see this simply divide by $c$ the weights of the star packing claimed by the lemma: the resulting fractional star packing shows that star cover rate of $G$ is at most $2-1 / c$.

Lemma 3.1: Let $G$ be a tree with at least 2 vertices, and suppose each core of $G$ has size at most $c$. Then there exists a star packing in $G$ so that (i) all edges are covered exactly $c$ times, and (ii) all vertices are covered at most $2 c-1$ times.

Proof: We replace each undirected edge $(u, v)$ of $G$ by $c$ directed edges between $u$ and $v$; the number of edges in each direction will be specified later. 
To obtain the star packing we partition the (now directed) edges into stars in such a way that all edges will be directed outward from the center of the star. Thus all outgoing edges from a vertex $v$ must be part of stars centered at $v$. Clearly, we can do this with as many stars centered at $v$ as the maximal number of outgoing edges from $v$ to some neighboring vertex. Furthermore $v$ will be a non-center vertex of exactly as many stars as the total number of incoming directed edges to $v$. The sum of these two numbers gives the total number of stars covering $v$. As there are exactly $c$ directed edges along each original edge, this cover number is $c$ plus the total number of incoming directed edges except the smallest number of incoming directed edges from a single neighbor.

Thus it suffices to show that we can direct these multiple edges so that this latter sum is at most $c-1$.

We start with assigning positive integers - weights - to each vertex. The weight of a set of vertices is the sum of the weights of the vertices in the set. Assigning weights is a technical step to ensure each vertex is in a maximum weight core.

Let $\mathcal{W}$ be the set of all positive integer weight functions making the weight of every core at most $c$. As each vertex is an element of some core, $\mathcal{W}$ has finitely many elements. Furthermore $\mathcal{W}$ is not empty: if every vertex has weight 1 , then by the definition of $c$, every core has weight $\leq c$. We call a weight function $w \in \mathcal{W}$ maximal if increasing $w$ by one at any one vertex yields a function outside $\mathcal{W}$. Clearly, a maximal weight function must exist in $\mathcal{W}$.

From now on fix such a maximal weight function $w \in \mathcal{W}$. The maximality of $w$ implies that for every vertex $v$ there exists a core containing $v$ whose weight is exactly $c$.

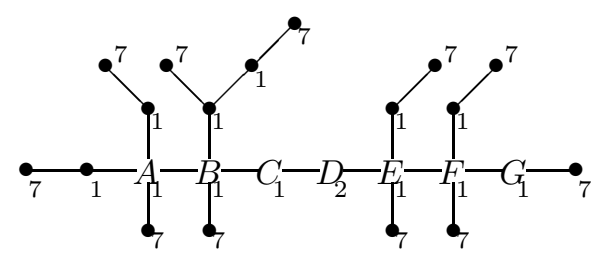

Fig. 2. A tree with weights and maximal core size $c=7$.

Now let $\left(v_{1}, v_{2}\right)$ be an edge of $G$. If either $v_{1}$ of $v_{2}$ is a leaf, then direct all $c$ edges between $v_{1}$ and $v_{2}$ toward the leaf. (If both $v_{1}$ and $v_{2}$ are leaves, then $G$ is a single edge, and there is nothing to prove.)

If neither $v_{1}$ nor $v_{2}$ is a leaf, then removing the edge $\left(v_{1}, v_{2}\right)$ splits $G$ into two disjoint subtrees, $G_{1}$ and $G_{2}$ where $G_{i}$ contains $v_{i}$. Let $C_{i}$ be a maximal weight (using the weight function $w$ ) core in $G_{i}$ such that $C_{i}$ contains $v_{i}$ and let its weight be $c_{i}=w\left(C_{i}\right)$. As $C_{1} \cup C_{2}$ is a core of weight $c_{1}+c_{2}$ in $G$, and all cores in $G$ has weight $\leq c$, we have $c_{1}+c_{2} \leq c$. Among the $c$ directed edges between $v_{1}$ and $v_{2}$ direct $c_{1}$ from $v_{1}$ towards $v_{2}$, and $c_{2}$ from $v_{2}$ towards $v_{1}$. If $c_{1}+c_{2}<c$ then direct the rest of these edges arbitrarily.

The tree depicted on figure 2 has maximal core size $c=7$, and the numbers show a maximal weight function. Each edge is replaced by seven directed edges, and the numbers the above procedure gives are

$$
\begin{array}{cccccc}
A \rightarrow B & B \rightarrow C & C \rightarrow D & D \rightarrow E & E \rightarrow F & F \rightarrow G \\
3 & 6 & \geq 1 & 2 & 4 & 6 \\
A \leftarrow B & B \leftarrow C & C \leftarrow D & D \leftarrow E & E \leftarrow F & F \leftarrow G \\
4 & 1 & \geq 2 & 5 & 3 & 1
\end{array}
$$

For example, when the edge $C D$ is deleted, the only core in the remaining graph containing $D$ is the singleton $\{D\}$ with weight 2 . This gives the value $\geq 2$ to $C \leftarrow D$ and similarly we have $\geq 1$ for $C \rightarrow D$. This leaves 4 more edges between $C$ and $D$ that we can direct arbitrarily. In all other edges in the above example we have $c_{1}+c_{2}=c$, thus the direction of all other edges are determined.

We claim that our construction satisfies the above requirement. Indeed, if $v$ is a leaf, then it has exactly $c$ incoming edges and no outgoing edge. Otherwise let $v$ be a non-leaf vertex, and $C$ be a core of maximal weight (according to $w$ ) containing $v$. By the maximality of $w, C$ has weight $c$. When deleting $v$ from $C$ each connected component of the remaining graph contains exactly one neighbor of $v$ in $C$. Let $v_{1}, v_{2}, \ldots$, $v_{s}$ be these neighbors and let $C_{i}$ be the connected component of $C-v$ containing $v_{i}$. Then

$$
c=w(C)=w(v)+w\left(C_{1}\right)+\cdots+w\left(C_{s}\right) .
$$

Both $C$ and $C-C_{i}$ are cores in $G-v v_{i}$ and they were considered when directing the edges along $v v_{i}$. Therefore we have at least $w\left(C_{i}\right)$ edges directed from $v_{i}$ to $v$ and at least $w\left(C-C_{i}\right)=c-w\left(C_{i}\right)$ edges going from $v$ to $v_{i}$. As this accounts for all $c$ edges between $v$ and $v_{i}$ these are the exact number of edges going either way. Thus the total number of incoming edges to $v$ from vertices in $C$ is

$$
w\left(C_{1}\right)+\ldots+w\left(C_{s}\right)=c-w(v) \leq c-1 .
$$

We have two cases: either $v$ has a leaf neighbor, or it has none. In the first case all non-leaf neighbors of $v$ are in $C$, as $C$ was chosen to be maximal. There are no incoming edges from leaves, thus in this case we are done.

In the other case no neighbor of $v$ is a leaf. Again by maximality all but one of the neighbors of $v$ must be in $C$. Let $v^{*}$ be the exceptional neighbor of $v$ outside $C$. Now $C-C_{i}$ is a core in the graph $G-v v^{*}$ and it contains $v$, thus at least $w\left(C-C_{i}\right)=c-w\left(C_{i}\right)$ edges are directed from $v$ toward $v^{*}$. It means that that the number of incoming edges from $v^{*}$ cannot be more than $w\left(C_{i}\right)$, which is the number of incoming edges from $v_{i}$. It shows that the smallest number of incoming edges come from $v^{*}$, and the total number of incoming edges from the other neighbors is at most $c-1$, which was to be shown.

\section{Algorithms}

We turn to the algorithmic part of Theorem 1.3 . Let $G$ be a tree. The size $c(G)$ of the maximal core in $G$ can be found by the following algorithm.

Pick an arbitrary root $r$ in $G$. For each vertex $v$ in $G$ let us denote by $G_{v}$ the subtree of $G$ "below" v, i.e., $G_{r}=G$ and for $v \neq r$ we obtain $G_{v}$ by deleting the edge connecting $v$ to its "parent" (the neighbor closer to $r$ ) and taking the connected component of $v$. 
First we order the the vertices in reverse breadth first search order (starting from the vertices farthest from the root) and compute the value $c(v)$ of the size of the largest core in $G_{v}$ containing $v$. We define $c(v)=0$ for leaf vertices $v$. If $v$ is not a leaf, then $c(v)$ is one plus the sum of $c\left(v_{i}\right)$ for all children $v_{i}$ of $v$ with the smallest summand left out of the summation. This enables us to compute $c(v)$ in time $O\left(d_{v}\right)$ from the values computed earlier. Here $d_{v}$ stands for the degree of $v$. This makes for a linear time algorithm for computing all the values $c(v)$.

Having computed $c(v)$ for each vertex, computing $c(G)$ is simple. If the largest core contains the root $r$, then its size is $c(r)$. Otherwise if $v \neq r$ is its vertex closest to the root its size is one plus the sum of $c\left(v_{i}\right)$ for all the children $v_{i}$ of $v$ (this time no summand is left out). Computing these values and finding the maximum takes linear time again.

Finally in order to construct the optimal secret sharing scheme one has to find a maximal weight function $w \in \mathcal{W}$. Notice that for an arbitrary weight function $w$ one can compute all the values $c_{w}(v)$ in linear time the same way we computed $c(v)$. Here $c_{w}(v)$ is the maximal $w$-weight of a core in $G_{v}$ containing $v$. Now increasing the weight of the root $r$ by $c-c_{w}(r)$ we can ensure that no core has weight over $c$ but the root is contained in a core of weight $c$. Starting from the all 1 weight function and repeating this procedure for all vertices as roots we find a maximal weight function. This takes quadratic time (still OK as the output is huge), but we remark that with a more careful analysis (increasing the weight of vertices in a single breadth first search order after computing first $c(v)$ without weights) a maximal weight function can be also obtained in linear time.

From a maximal weight function $w$ one can orient $c_{w}(v)$ edges from $v$ to its parent $(v \neq r)$ and $c-c_{w}(v)$ edges from the parent to $v$. This yields an optimal star packing. Now we apply Stinson's technique [13] to obtain the secret sharing scheme on $G$ by combining linear schemes on the individual stars. The parameters of this combined scheme are as stated in Theorem 1.3 .

\section{REFERENCES}

[1] C. Blundo, A. G. Gaggia, D. R. Stinson: On the Dealer's Randomness Required in Secret Sharing Schemes Des. Codes Cryptogr. Vol 11(3) (1997), pp.235-260

[2] C. Blundo, A. De Santis, R. De Simone, U. Vaccaro: Tight bounds on the information rate of secret sharing schemes Des. Codes Cryptogr. Vol 11 (1997) pp.107-122

[3] C. Blundo, A. De Santis, L.Gargano, U. Vaccaro: On the information rate of secret sharing schemes, Advances in Cryptology - CRYPTO'92, Lecture Notes in Comput. Sci. 740 (1993) pp.148-167

[4] C. Blundo, A. De Santis, D. R. Stinson, U. Vaccaro: Graph decomposition and secret sharing schemes, J. Cryptology Vol 8 (1995) pp.39-64

[5] E. F. Brickell, D. M. Davenport: On the classification of ideal secret sharing schemes, J. Cryptology Vol 4 (1991) pp.123-134

[6] E. F. Brickell, D. R. Stinson: Some improved bounds on the information rate of perfect secret sharing schemes, J. Cryptology Vol 5 (1992) pp.153166

[7] R. M. Capocelli, A. De Santis, L. Gargano, U. Vaccaro: On the size of shares of secret sharing schemes, Journal of Cryptology, vol 6(1993), pp. $157-168$

[8] L. Csirmaz: Secret sharing schemes on graphs, Studia Mathematica, vol 44(3), pp 297-306, 2007

[9] M. van Dijk, T. Kevenaar, G. Schrijen, P. Tuyls: Improved constructions of secret sharing schemes by applying $(\lambda, \omega)$-decompositions, Inf. Process. Lett. vol 99(4), 2006, pp.154-157
[10] J. Martí-Farré, C. Padró: On secret sharing schemes, matroids and polymatroids, Proceedings of the 4th conference on Theory of Cryptography, Lecture Notes in Comput. Sci. 740 (2007) pp.273-290

[11] S. A. Plotkin, D. B. Shmoys, Eva Tardos: Fast Approximation Algorithms for Fractional Packing and Covering Problems Math. Oper. Res. Vol 20, pp 257-301, 1995

[12] Edward R. Scheinerman, Daniel H. Ullman: Fractional Graph Theory: A Rational Approach to the Theory of Graphs Wiley-Interscience, (1997)

[13] D. R. Stinson: Decomposition constructions for secret sharing schemes, IEEE Trans. Inform. Theory 40 (1994) pp 118-125.

[14] D. R. Stinson, R. Wei: Bibliography on Secret Sharing Schemes, available at http:// www.cacr.math.uwaterloo.ca/ rdstinson/ssbib.html

László Csirmaz has been with Central European University, Budapest, since 1996. Before that he worked as a researcher at the Rényi Institute of Mathematics, Budapest. His main research interests include secret sharing, Shannon theory, and combinatorial games.

Gábor Tardos received his $\mathrm{PhD}$ in mathematics from Eötvös University in 1988. A research fellow at the Reényi Institute, Budapest, Hungary since 1990. Canada Research Chair of Computational and Discrete Geometry at the Simon Fraser University, BC, Canada, since 2005.

His main research interests are combinatorics, discrete and computational geometry, and complexity theory. 